This document is the accepted manuscript version of the following article: Rainò, G., Utzat, H., Bawendi, M. G., \& Kovalenko, M. V. (2020).

Superradiant emission from self-assembled light emitters: from molecules to quantum dots. MRS Bulletin, 45(10), 841-848.

https://doi .org/10.1557/mrs.2020.250

\title{
Superradiant emission from self-assembled light emitters: From molecules to quantum dots
}

G. Rainò, H. Utzat, M.G. Bawendi, and M.V. Kovalenko

G. Rainò, ETH Zürich and Empa Dübendorf, Switzerland; rainog@ethz.ch

H. Utzat, Stanford University, USA; hutzat@stanford.edu

M.G. Bawendi, Massachusetts Institute of Technology, USA; mgb@mit.edu

M.V. Kovalenko, ETH Zürich and Empa Dübendorf, Switzerland;

mvkovalenko@ethz.ch

Colloidal synthesis methods and ultrahigh-vacuum molecular beam epitaxy can tailor semiconductor-based nanoscale single emitters - quantum dots - as the building blocks for classical optoelectronic devices, such as lasers, light-emitting devices (LEDs), and display technologies. These novel light sources retain basic resemblance with luminescent organic molecules, individually and in the aggregated forms. Highly ordered superstructures of quantum dots, obtained via scalable bottom-up self-assembly, exhibit diverse collective phenomena, such as band-like charge transport or superradiant emission. Superradiance emerges from coherent coupling of several emitters via a common radiation field resulting in a single giant dipole leading to short (sub-nanosecond) and intense (proportional to the squared number of coupled emitters) bursts of light. In this article, we review the basic principles and progress in the development of superradiant emitters with organic molecules and inorganic quantum dots, in view of their integration into classical and novel quantum light sources.

Keywords: quantum materials, quantum dot, photoemission, single-photon source/emitter, optoelectronic. 


\section{Introduction}

Light is a cross-disciplinary subject that has fascinated scientists for centuries and has revolutionized society through medicine, transoceanic communications, highspeed internet, and high-performance computing, just to mention a few examples. Disruptive progress in the development of light sources has been tightly linked to innovations in the nanoscale engineering of materials. In 2000, the Nobel Prize in physics was awarded jointly to H. Kroemer and Z.I. Alferov, for "developing semiconductor heterostructures used in high-speed-photography and optoelectronics,"1 a breakthrough that was enabled by the monolayer thickness control achieved in epitaxial growth methods. Likewise, efficient blue gallium nitride (GaN) light-emitting devices (LEDs) (I. Akasaki, H. Amano, and S. Nakamura, Nobel prize in Physics in $2014^{2}$ ) have become possible once the quality of GaN layers was much improved by introducing an AIN buffer layer, which mitigated the effect of the lattice mismatch with the substrate and prevented formation of defects and dislocations.

Quantum dots (QDs), ${ }^{3}$ nanometer-sized semiconductor crystals with confined electronic wavefunctions are outstanding light emitters with high efficiency and tunability in their emission energy. Colloidal synthesis of semiconductor nanocrystals (often called colloidal QDs) has undergone tremendous progress and become a viable alternative to fully solid-state growth pathways such as ultrahigh-vacuum molecular beam epitaxy (MBE) in terms of the electronic and optical materials quality. By virtue of the quantum confinement effect, ${ }^{4,5}$ a wide range of bandgap energies and hence colors can be obtained by simply controlling the QD size (Figure 1a). At present, compositional and morphological engineering with colloidal methods extend far beyond the capacity of $\mathrm{MBE}$ and similar methods for a broad range of semiconductors. Owing to their low synthesis costs, along with facile solution processability, colloidal QDs have made significant commercial inroads in the lighting ${ }^{6}$ and displays markets (Figure 1b).

In the realm of molecular light emitters, the synthesis toolbox of organic chemistry had been instrumental in adjusting the energy levels, such as tuning the 
singlet-triplet splitting, ${ }^{7-9}$ thereby boosting the development of organic lightemitting diodes (Figure 1b) ${ }^{10-12}$ for applications in lighting and TV displays. More recently, nanoscale control in materials growth has been pivotal for the generation of non-classical light, in particular, to yield a stream of single and indistinguishable photons. ${ }^{13,14}$ Contrary to other light sources, like lasers and LEDs whose light output can be modelled using classical electromagnetism theory, non-classical, quantum light emitters are characterized by temporal intensity correlations that cannot be explained through classical intensity fluctuations but required a full quantum-mechanical description (e.g. quantization of electromagnetic field). These quantum light sources open up new scenarios for quantum communication ${ }^{15}$ and quantum sensing applications.

Spontaneous emission (SE) of photons occurs owing to the coupling of an excited two-level system ([TLS] Figure 1c) to the vacuum modes of the electromagnetic field, effectively stimulated by its zero-point fluctuations. ${ }^{16}$ In solid-state light emitters, an effective TLS can be realized by means of optical or electrical excitation, generating electrons and holes which then recombine by emitting photons. When the radiative process is a sole channel for relaxation of an excited state, the emission band is extremely narrow and only limited by the lifetime (denoted $\mathrm{T}_{1}$ ) of the radiative transition (Fourier-transform limit, $\Delta \mathrm{E}=1$ / $\mathrm{T}_{1}$ ). This is usually observed when optically allowed atomic transitions are probed in ultrahigh vacuum. ${ }^{15}$ However, for solid-state emitters, the fluorescence typically originates from excitons, which are quasi-particles susceptible to spectral changes induced by the solid-state environment (Figure 1c). Variations in the size and/or composition from particle to particle usually broaden the photoluminescence (PL) of an ensemble of QDs, in a similar way conformational disorder does in an ensemble of organic molecules, leading to inhomogeneous emission broadening. Thermally activated crystal vibrations, often called phonons, or nuclear spin fluctuations coupling to the angular momentum of the exciton, can broaden the emission lines in single emitters significantly (homogeneous broadening) (Figure 1c). ${ }^{17}, 18$ Both homogeneous and inhomogeneous broadening activate a process known as dephasing, which 
determines how long a system will maintain its own 'coherence', or its internal memory of the oscillating dipole phase (Figure 1c). In organic molecules, dephasing is determined by dynamic intermolecular interactions, and measurements of the dephasing time $\left(\mathrm{T}_{2}\right)$, the characteristic timescale on which the ensemble of emitters loses memory of the emitted light's phase, can therefore provide information on the interaction and coupling between the excited state in a molecule and its environment.

Ultimate control over the luminescence of materials requires precise control of inhomogeneous and homogeneous broadening. Colloidal QDs have undergone great advances in this regard; for instance, colloidal QDs can now be synthesized with extremely low size dispersion, thus largely eliminating inhomogeneous broadening. The emission spectrum of an ensemble of QDs is then similar to that of an isolated particle. ${ }^{19,20}$ Addressing the coupling with phonons and nuclear spins and the resulting dephasing has turned to be far more complex, and is the subject of intense research. ${ }^{17,21}$ Cooling the material down to cryogenic temperatures is still the most effective path to the deactivation of phonons, allowing the observation of new phenomena, such as superradiant (SR) emission. $^{22}$

In 1954, Dicke predicted that an ensemble of $N$ two-level systems confined in a volume smaller than $\sim \lambda^{3}$ ( $\lambda$ is the corresponding emission wavelength of the TLS) can exhibit coherent and cooperative spontaneous emission. ${ }^{23}$ This so-called SR emission results from the coherent coupling between individual TLSs through the common vacuum modes, leading to a macroscopic optical polarization. From a classical point of view, the collective emission is equivalent to coupled and synchronized (phase-locked) pendula (Figure 2a). The resulting light output can be much more intense than the sum of the individual emitters, similarly to the occurrence of constructive interference between two optical waves. It only occurs, however, when the emitters fulfill stringent requirements, such as high coupling strength to the light field manifested in a short radiative lifetime $\left(\mathrm{T}_{1}\right)$, comparatively long coherence time $\left(\mathrm{T}_{2}\right)$ and the same emission energy. The superradiant emission is characterized by a 
cooperative accelerated decay time $\tau_{\text {coupled }}=\frac{\tau_{\text {uncoupled }}}{N}$, that is inversely proportional to the number of coupled emitters $N$. As a result of this shortening in the radiative lifetime, the photoluminescence peak intensity increases proportionally to $N^{2}$, giving rise to very bright emission (Figure $2 \mathrm{~b}$ ). These compelling features could potentially boost the device performance (e.g., ultrabright LEDs with sub-nanosecond switching time) could be developed with applications, for instance, in visible light communication (VLC) networking and sensing.

Light is often characterized simply by its classical properties, such as intensity or coherence. However, exploring its quantum properties, described by photon statistics, can give further insights into the processes governing light emission. For the present case of superradiant emission, the quantum-mechanical inter-emitter correlations and the underlying physics of the collective decay, dramatically change the photon statistics of the emitted light. A simple way to test photon statistics and whether the emitted light field propagates on packets of one or multiple photons is to perform the Hanbury Brown and Twiss experiment. A sketch of the experimental setup is presented in Figure 2c. The emitted light is sent to a 50:50 beam-splitter and reaches two single-photon detectors (D1 and D2 in Figure 2c). The output of detectors is sent to correlation electronics that measures the time delay between coincident detection events, or in other words, the probability of detecting a photon on both detectors as a function of the time delay between them. An isolated TLS (Figure 2d) must exhibit a strong dip at zero-delay time in the second-order intensity-correlation function $(\mathrm{g} 2(\mathrm{t}))$, if a stream of single photons with well-defined temporal delay between successive photons is to be generated. This is because when a single photon is present, the probability that both detectors fire at zero delay time is null. This is the so-called anti-bunching behavior, commonly observed, for example, in studies of photon statistics of isolated single molecules.$^{13,14}$ With synchronization and coherent coupling among multiple emitters, strongly time-correlated photons may emerge (Figure 2d), which give rise to a bunching peak in the second-order correlation 
function. ${ }^{24}$ Photon statistics has become the hallmark method to study quantum light, and it has also been widely applied to superradiant quantum emitters.

Early experiments by Skribanowitz et al. demonstrated SR in dense HF gases, ${ }^{25}$ followed by reports on SR in solid-state systems, such as $\mathrm{CuCl}$ nanocrystals embedded in a $\mathrm{NaCl}$ matrix,${ }^{26} \mathrm{O}_{2}^{-}$-doped $\mathrm{KCl}$ crystals, ${ }^{27}$ and epitaxially-grown InGaAs quantum wells. ${ }^{22,28}$ In this overview article, we analyze the state-of-the-art of two technologically relevant classes of nanomaterials, organic molecules and quantum dots, as superradiant emitters. We highlight how self-assembly of these emitters could be a key pathway for future developments of SR emitters, with potentials in classical light emitting devices and in quantum light sources.

\section{Molecular aggregates}

For almost eighty years after their serendipitous discovery, ${ }^{29}$ molecular aggregates, assemblies of molecules with well-defined molecular packing, have continuously excited scientists across many disciplines because the cooperative emission among molecules in the aggregates significantly alters their optoelectronic properties, beyond what can be achieved by engineering the individual molecules. The most widespread model used to understand how molecular packing impacts the optical properties was formulated by Kasha. ${ }^{30}$ In Kasha's framework, the coulombic coupling between two molecules, as determined by the alignment of their transition dipoles, the complex vector of the electric-field associated with the transition between the two states, induces energetic shifts in the main absorption peak and changes in the radiative decay rates when compared to uncoupled molecules. There are two main forms of molecular aggregates, historically defined as $\mathrm{H}$ - and $\mathrm{J}$-aggregates, with very different molecular packing. Exquisite control of the supramolecular selforganization packing can be obtained by judicious selection of the solvent/additives and the dye concentration. ${ }^{29}$ In $\mathrm{H}$-aggregates, the transition dipole moments align "side-by-side" leading to a spectral blue-shift and suppressed radiative decay rate, while in J-aggregates, the transition dipole 
moments align "head-to-tail" leading to a spectral red shift and an enhanced radiative decay rate (Figure 3a). ${ }^{31}$

An example of how the optical properties are transformed by the supramolecular architecture is reported in Figure $3 b .{ }^{32}$ Contrary to the absorption spectrum of isolated molecules, which is rather broad and peaked around $520 \mathrm{~nm}$, for the specific case of carbocyanine dye, the formation of an aggregate strongly narrows the absorption band, which now appears at longer wavelengths $(590 \mathrm{~nm})$. This narrowing is due to the strong exciton delocalization over many coherently coupled molecules, which increases the absorption cross section and reduces exciton-phonon coupling. As predicted by Dicke, the radiative lifetime decreases owing to coherent coupling between multiple molecules (up to hundreds), resulting in decay times down to tens of picoseconds at cryogenic temperatures (Figure $3 \mathrm{c}$ ). ${ }^{32} \mathrm{By}$ increasing the temperature, the lifetime progressively increases, as exciton-phonon coupling localizes the excitation, resulting in coherence volumes significantly smaller than the physical size of the aggregates.

Although molecular aggregates are the longest-known superradiant material system, a consolidated picture concerning the emitted photon statistics is still missing. We could not find reports on photon statistics of J-aggregates, likely owing to the fragile nature of the aggregates hampering the spectroscopy of single domains in aggregates. In Figure 3d, the photon statistics of isolated and aggregated molecules is shown for conjugated polymer chains. ${ }^{33}$ The antibunching dip (a measure of the single photon purity) is more pronounced in the aggregate form compared to the single molecule. This counterintuitive observation is a result of a more efficient electron-electron annihilation in the aggregate. However, cooperative emission and bunching in the second-order correlation have also been reported. For example, for two molecules that were separated by ca. $12 \mathrm{~nm}$, tuning the molecules into resonance via the application of an electric field drastically changes the photon statistics from anti-bunching to bunching (Figure 3e), confirming the occurrence of coherent coupling and superradiant emission. ${ }^{34}$ 


\section{Epitaxially grown quantum dots}

Epitaxial QDs, such as $\operatorname{In}(\mathrm{Ga})$ As QDs in a GaAs matrix, are typically produced through the Stranski-Krastanov growth method that makes use of the 7\% latticeconstant mismatch between GaAs and InAs. This mismatch induces a lattice strain distribution that causes a transition from two-dimensional planar layers to three-dimensional (3D) islands at InAs thicknesses of ca. 1.7 monolayers. The islands are typically $10-30 \mathrm{~nm}$ in lateral size and 2-5 $\mathrm{nm}$ out of plane, with a typical lens-shape profile. Subsequent overcoating with epitaxial GaAs imparts $3 \mathrm{D}$ quantum confinement, seen as the emergence of discrete optical transitions at cryogenic temperatures with the lowest transitions in the 900-1000 nm wavelength range. Epitaxial QDs can be easily integrated in photonic structures, e.g. micro lenses and photonic crystals, ensuring a very high photon extraction efficiency. (Figure 4a). ${ }^{35-37}$

At present, epitaxial QDs are among the best solid-state sources of ondemand single photons and entangled photon pairs. ${ }^{14}$ QDs exhibit small dephasing rates leading to long optical coherence times when resonantly pumped, allowing the generation of highly indistinguishable single photons. However, because strain drives the formation of QDs, they have random spatial positions and are still inhomogeneously broadened due to QD size distributions and local environmental variations. Minimizing the energetic disorder has been the main roadblock for the occurrence of coherent coupling among several QDs. ${ }^{24,36}$

Recently, a solution to tune the emission energy of a QD was proposed by using compressive strain: ${ }^{37}$ the QD spectral lines could be tuned over a spectral range of several tens of $\mathrm{meV}$, exceeding the inhomogeneous broadening of QD ensembles. Moreover, a precision of ca. $1 \mu \mathrm{eV}$ could be achieved, narrower than the single QD homogeneous linewidth (Figure 4b). This technique allows virtually any arbitrary QD to be tuned to a precise target wavelength, without affecting optical quality. This potentially scalable method ensures several QDs, presently up to three, to be tuned to the same resonance wavelength. By performing second-order correlation measurements, the occurrence of cooperative emission can be demonstrated (Figure 4c-d). In Figure 4c, photon correlation in 
the case of two QDs is presented for different emission detuning energies $(\delta)$. For two distinguishable single photon emitters $(\delta=46 \mu \mathrm{eV})$, the second-order correlation function exhibits an anti-bunching dip of 0.5 at $\tau=0$. This is expected for two uncoupled QDs with pure single-photon emission. For two resonant emitters $(\delta=0 \mu \mathrm{eV})$, there is a sharp bunching peak emerging around $\tau=0$, which is a clear signature of superradiance. In Figure 4d, three QDs were tuned into resonance. First, when only one QD was measured, a typical anti-bunching curve with a minimum close to zero was observed. Two of the QDs are then tuned into resonance and measured, showing a reduction in the anti-bunching dip and a clear central peak. Finally, when all three QDs are tuned into resonance, the antibunching dip becomes shallow and the central peak amplitude increases. Coupling among a much larger number of QDs could be an important future endeavor for the realization of large, massively quantum entangled states, further boosting our fundamental understanding in many-body light-matter interactions.

\section{Perovskite quantum dots}

Colloidal QDs of perovskite-type lead halides $\left(\mathrm{APbX}_{3}, \mathrm{~A}=\mathrm{Cs}\right.$, formamidinium; $\mathrm{X}=\mathrm{Cl}, \mathrm{Br}, \mathrm{I})$ offer bright, tunable, narrow band room-temperature luminescence tunable over the whole visible wavelength range by adjusting the halide composition $\left(\mathrm{Cl} / \mathrm{Br}\right.$ and $\mathrm{Br} / \mathrm{I}$ solid solutions) and size $(3-15 \mathrm{~nm}) \cdot{ }^{38-41}$ Unlike earlier generations of colloidal QDs (metal chalcogenides and pnictides), perovskite QDs exhibit room-temperature photoluminescence quantum yields of up to unity without additional electronic surface passivation. This is a manifestation of the widely observed defect tolerance of perovskite semiconductors, that is, the (near) absence of detrimental mid-gap states, ubiquitously arising in other semiconductors owing to the dangling bonds at crystal defects, surfaces and interfaces. ${ }^{42}$ Spectroscopy of single perovskite QDs has revealed narrow-band (ca. $90 \mathrm{meV}$ at $\mathrm{RT},{ }^{20}$ less than $1 \mathrm{meV}$ at $5 \mathrm{~K}^{43}$ ) and blinking-free emission with high oscillator strengths. ${ }^{44}$

A recent study revealed the origin of the high oscillator transition in perovskite materials as being due to a bright triplet state with orthogonal dipole orientations (Figure 5a). ${ }^{45}$ This peculiarity, not observed in other II-VI or III-V 
QDs can facilitate the appearance of collective emission by enabling QD coupling in all spatial dimensions (omnidirectional coupling). Larger QDs ( $\geq 8 \mathrm{~nm})$, which are in a weak confinement regime (particle size similar to the exciton Bohr diameter), exhibit low energetic disorder ${ }^{20}$ and giant oscillator strength transition, ${ }^{45}$ which translates into short radiative lifetime on the order of 200-300 ps (Figure 5b). ${ }^{46}$ Such fast emission contrasts strikingly with those of colloidal IIVI QDs or organic molecules, which are orders of magnitude slower.

Furthermore, photon correlation Fourier spectroscopy of single perovskite QDs revealed a long coherence time of ca. $80 \mathrm{ps}$ as well as minimal spectral fluctuations over time. ${ }^{46}$ Together, the combination of fast radiative lifetimes and long coherence times are important prerequisites for efficient optical coupling in superlattices of perovskite QDs.

Monodisperse perovskite QDs can be assembled into highly ordered superlattices upon controlled solvent evaporation (see inset of Figure $5 \mathrm{c}$ ). ${ }^{47}$ The PL spectrum from such assemblies at cryogenic temperatures is composed of two bands, with the redshifted band being associated with coupled QDs (Figure 5c). ${ }^{47}$ Time-resolved spectroscopy has shown much faster radiative decay for this lowerenergy band (Figure 5d), with radiative lifetimes decreasing to $20 \mathrm{ps}$ at high excitation densities. Given the relationship between the radiative lifetime and the total number $N$ of coupled QDs, an estimation of more than 10 coupled QDs could be made. Remarkably, oscillations in the time domain, often referred to as Burnham-Chiao ringing behavior, attest the onset of superfluorescence, a specific case of collective phenomena in which the dipoles self-lock into a macroscopic dipole ${ }^{47}$ after being photo-excited. We note that this superfluorescence is distinct from superradiance in J-aggregates where the coherent coupling among the molecules is already present in the electronic ground state. Second-order correlation measurements (see inset of Figure 5d) corroborate the conclusion that collective emission shows a bunching peak with amplitudes larger than two, demonstrating the feasibility of generating higher photon-number states with perovskite QDs. ${ }^{47}$ 


\section{Summary and outlook}

After a short introduction on the basic principles governing collective radiative processes, we have reviewed the present state of research in two technologically important classes of quantum emitters, namely organic molecules and inorganic quantum dots, from the perspective of engineering superradiant emission. We discussed the spectroscopic evidences and possible applicability of collective emitters as bunched-photon sources. The superradiance in the materials discussed here, if realized at room temperature, could increase the efficiency of lightemitting devices significantly. However, superradiance in these commonly used light emitters still requires cryogenic temperatures thus far, hampering the direct application of the concept in devices.

Translation to room-temperature operation, however, presents a formidable scientific challenge, which requires a better understanding of the dephasing processes in solid-state emitters and methods to control them. Here, the $\mathrm{NV}$ center in diamond ${ }^{48,49}$ could inspire the design of novel quantum emitters with improved inter-emitter coupling for boosted light emission. The reduced exciton-phonon coupling tied to the symmetry of the defects leads to overall small dephasing rates in NV centers, which have been shown to exhibit roomtemperature $\mathrm{rSR}^{48}$

Just like single quantum emitters have revolutionized microscopy by the introduction of superlocalization methods with nanoscale (subdiffraction) resolution, ${ }^{50} \mathrm{~N}$-photon bundles ${ }^{51}$ will impact novel quantum imaging and quantum communication schemes, with potential also in the quantum computing domain (boson sampling). ${ }^{52,53} \mathrm{We}$ hope to convince the reader that selfassembled quantum emitters with coherent interactions between these emitters present a clear path forward for exploring the generation of high-number photon states. $^{51}$

\section{Acknowledgments}

H.U. was funded by the US Department of Energy, Office of Basic Energy Sciences, Division of Materials Sciences and Engineering (Award No. DE-FG0207ER46454). M.V.K. and G.R. acknowledge financial support from the European 
Union through Horizon 2020 (ERC Consolidator Grant SCALE-HALO, Grant Agreement No. 819740).

a

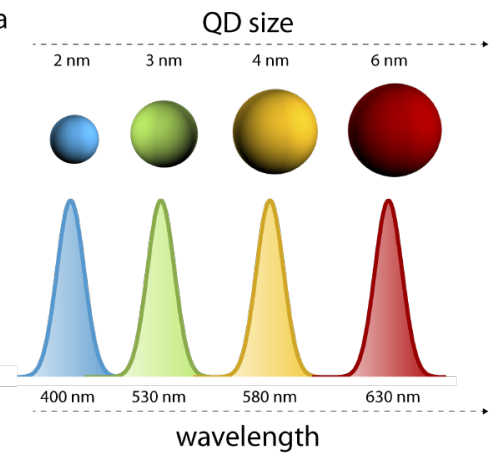

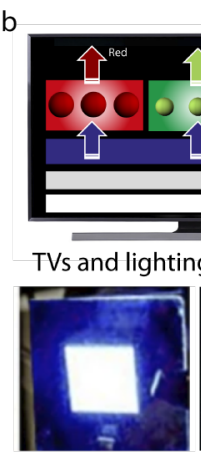

OLED

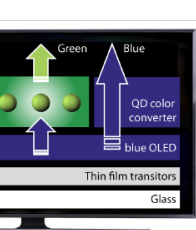

gapplications

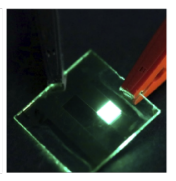

QDLED

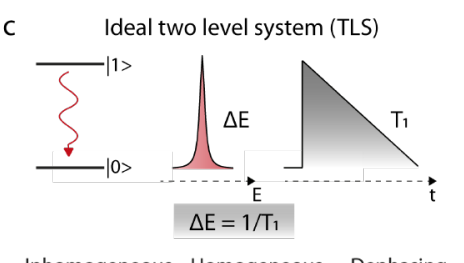

Inhomogeneous Homogeneous Dephasing

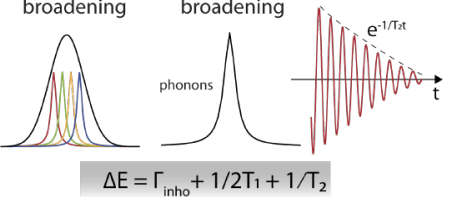

Figure 1. Functional nanomaterials: main applications and basic principles of spontaneous emission: (a) Sketch of the size-dependent emission properties of colloidal QDs: the quantum confinement effect. (b) Examples of emissive nanomaterials for TV displays and organic and quantum dot-based light emitting diodes (OLEDs and QDLEDs, respectively). (c) Basic principles governing the spontaneous emission process, inhomogeneous and homogeneous emission broadening and dephasing. (b) Adapted with permission from Reference 12 Nature Publishing Group. 
a

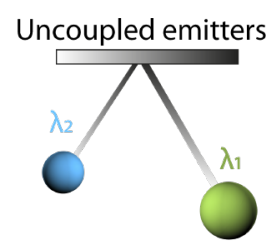

Energetic disorder

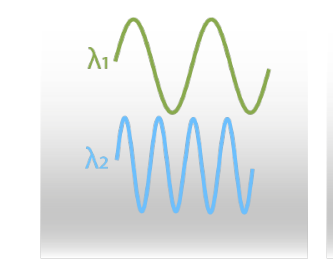

C

Hanbury Brown and Twiss experiment

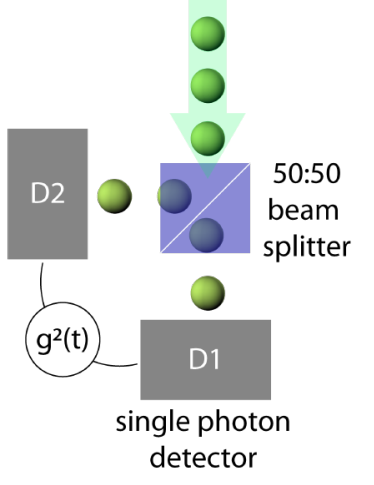

b

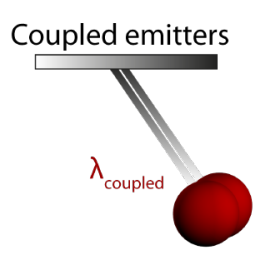

Coherence

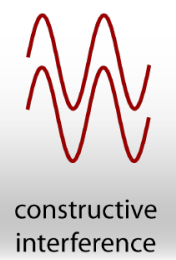

d single dipole

superradiant dipoles
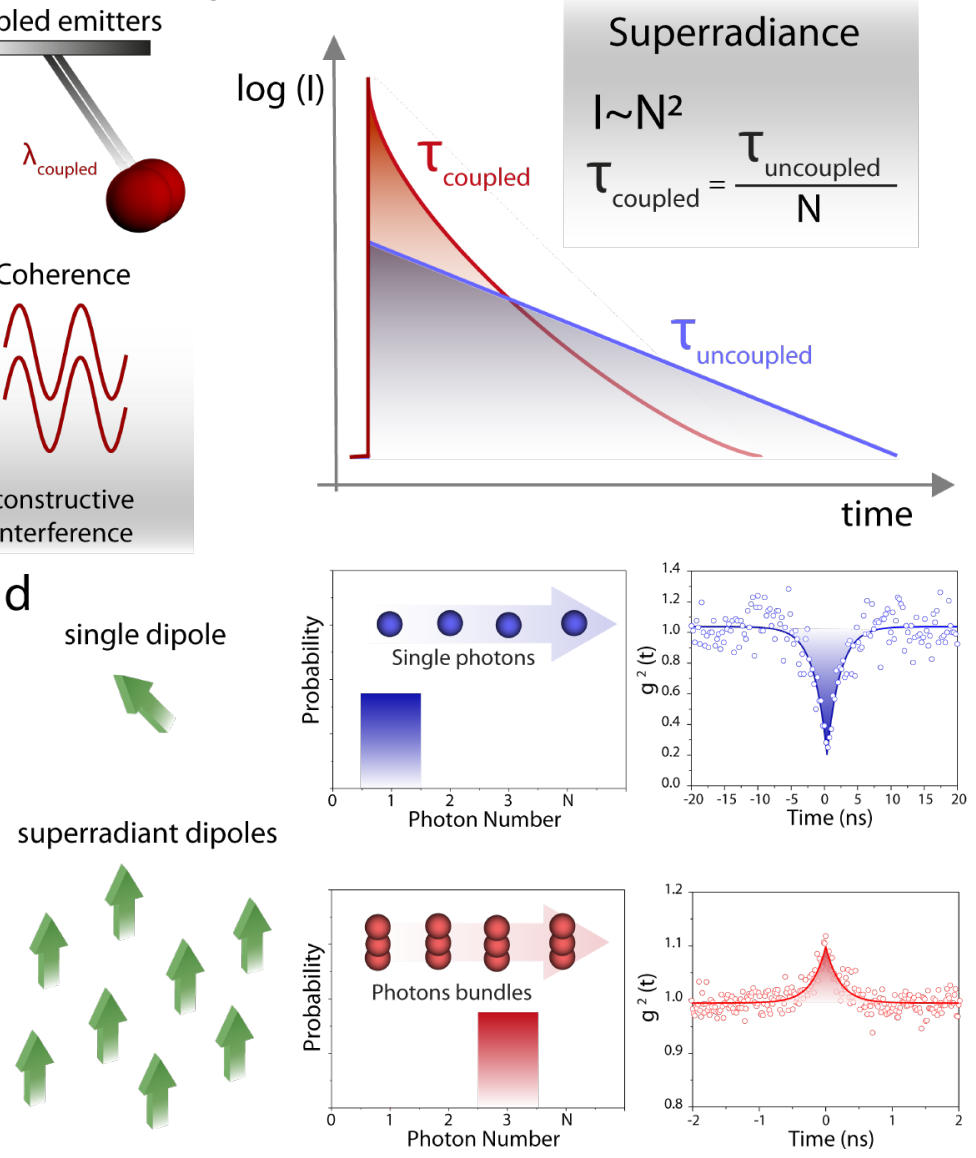

Figure 2. Collective superradiant emission: accelerated radiative lifetime and photon statistics. (a) Classical representation of uncoupled and coupled emitters given by two oscillating pendula; due to fast dephasing and energetic disorder, uncoupled emitters oscillate out of phase. Coupled emitters, however, can phaselock (via the common photon field) and behave as a single, coherent pendulum. (b) Superradiance induces an accelerated radiative decay, inversely proportional to the number $N$ of coherently coupled emitters. As a consequence, the PL peak intensity scales as $N^{2}$. (c) Schematic of the Hanbury Brown-Twiss interferometer. (d) Isolated and coupled (superradiant) dipoles with the expected photon statistics, changing from anti-bunching to bunching, respectively. 
a
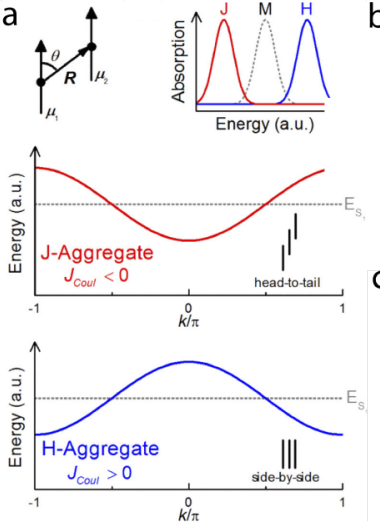

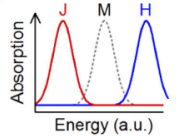

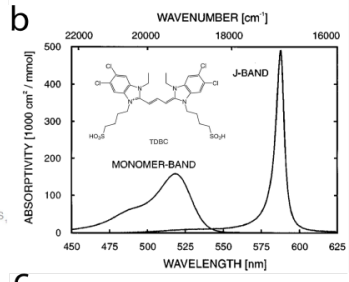
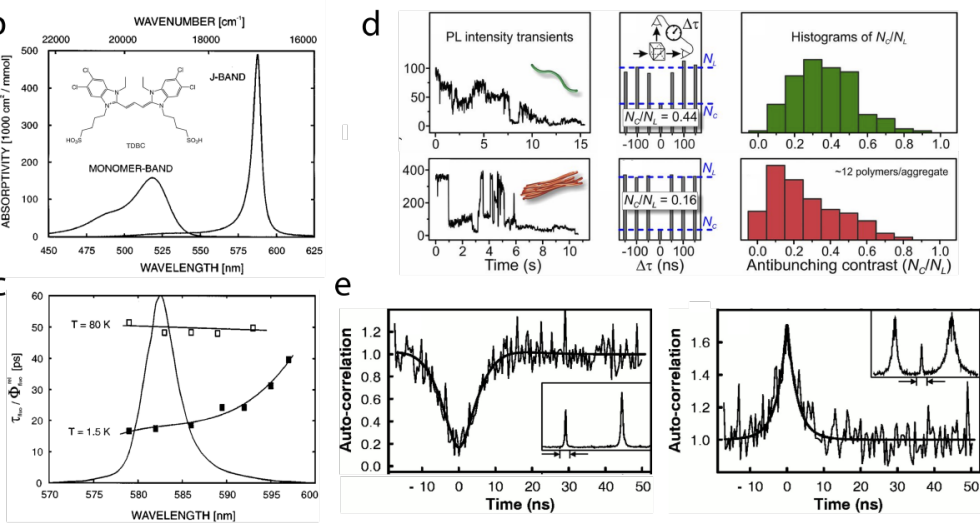

Figure 3. Superradiant emission: the case of molecular J-aggregates. (a) Transition dipole geometry for evaluating coulombic coupling under the point dipole approximation. In $\mathrm{J}$ - and $\mathrm{H}$-aggregates, the optically allowed exciton $(\mathrm{k}=$ 0 ) resides at the bottom and top of the band, respectively. (b) Monomer band (broad with peak around $520 \mathrm{~nm}$ ) and J-aggregate band (narrow with peak around $590 \mathrm{~nm})$ of TDBC (5,5',6,6'-tetrachloro-1, 1'-diethyl-3,3'-di(4-sulfobutyl)benzimidazolocarbocyanine) molecules. (c) Wavelength dependence of the fluorescence lifetime of TDBC aggregates at $1.5 \mathrm{~K}$ and $80 \mathrm{~K}$. (d) Photon antibunching from single and multichain aggregates. Due to efficient interchain energy transfer and singlet-singlet annihilation, the anti-bunching dip is more pronounced in the aggregate state. (e) Two individual fluorescent molecules separated by $12 \mathrm{~nm}$ in an organic crystal can undergo a strong coherent dipoledipole coupling and displays photon bunching resulting from the collective emission of two photons with higher probability than expected from random photon statistics. (a) Adapted with permission from Reference 31. (b, c) Adapted with permission from Reference 32. (d) Adapted with permission from Reference 33. (e) Adapted with permission from Reference 34. 
a

C
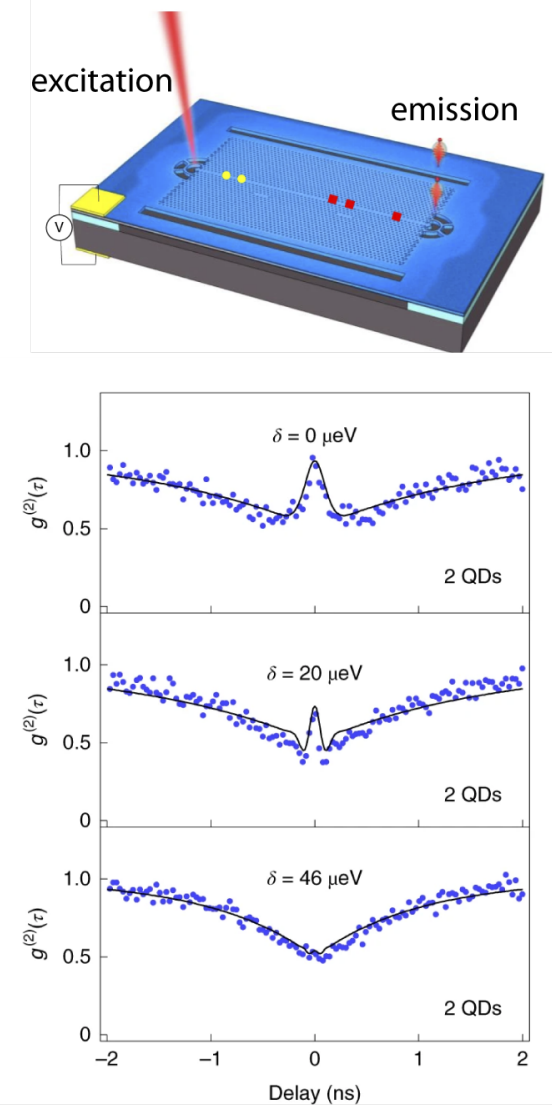

b
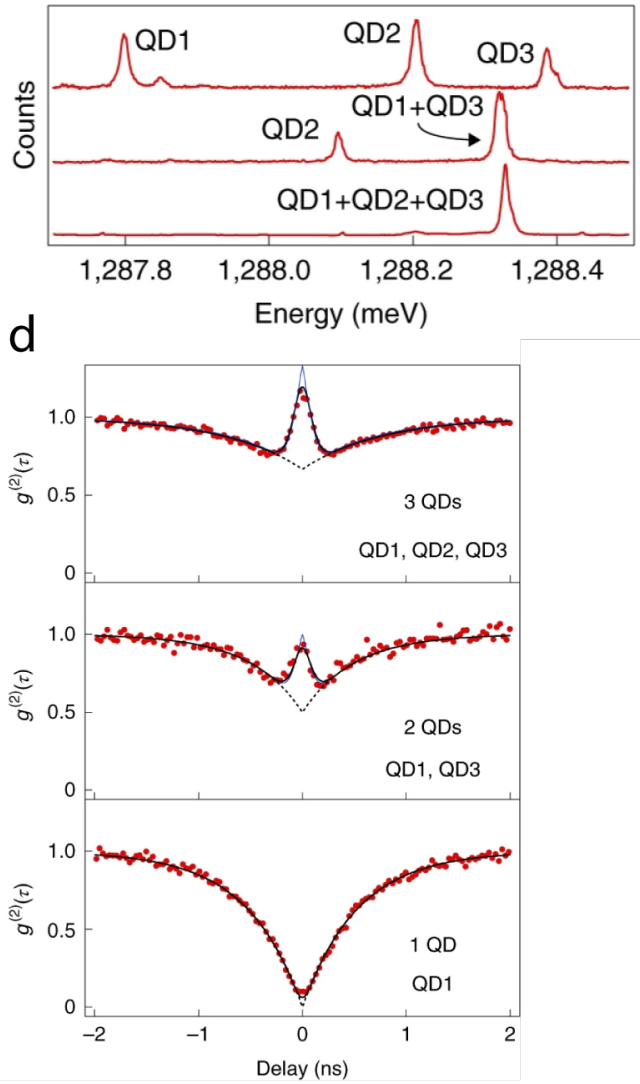

Figure 4. Superradiant emission: the case of epitaxially grown quantum dots. (a) SEM image of a photonic crystal waveguide and schematic with the positions of the QDs indicated with colored points. (b) Emission spectrum showing the PL of three QDs. (c) Second-order intensity-correlation measurements for resonant and detuned QDs. (d) Second-order photon correlation measurements for one (bottom), two (middle), and three (top) resonant emitting QDs. (a) Adapted with permission from Reference 37. 

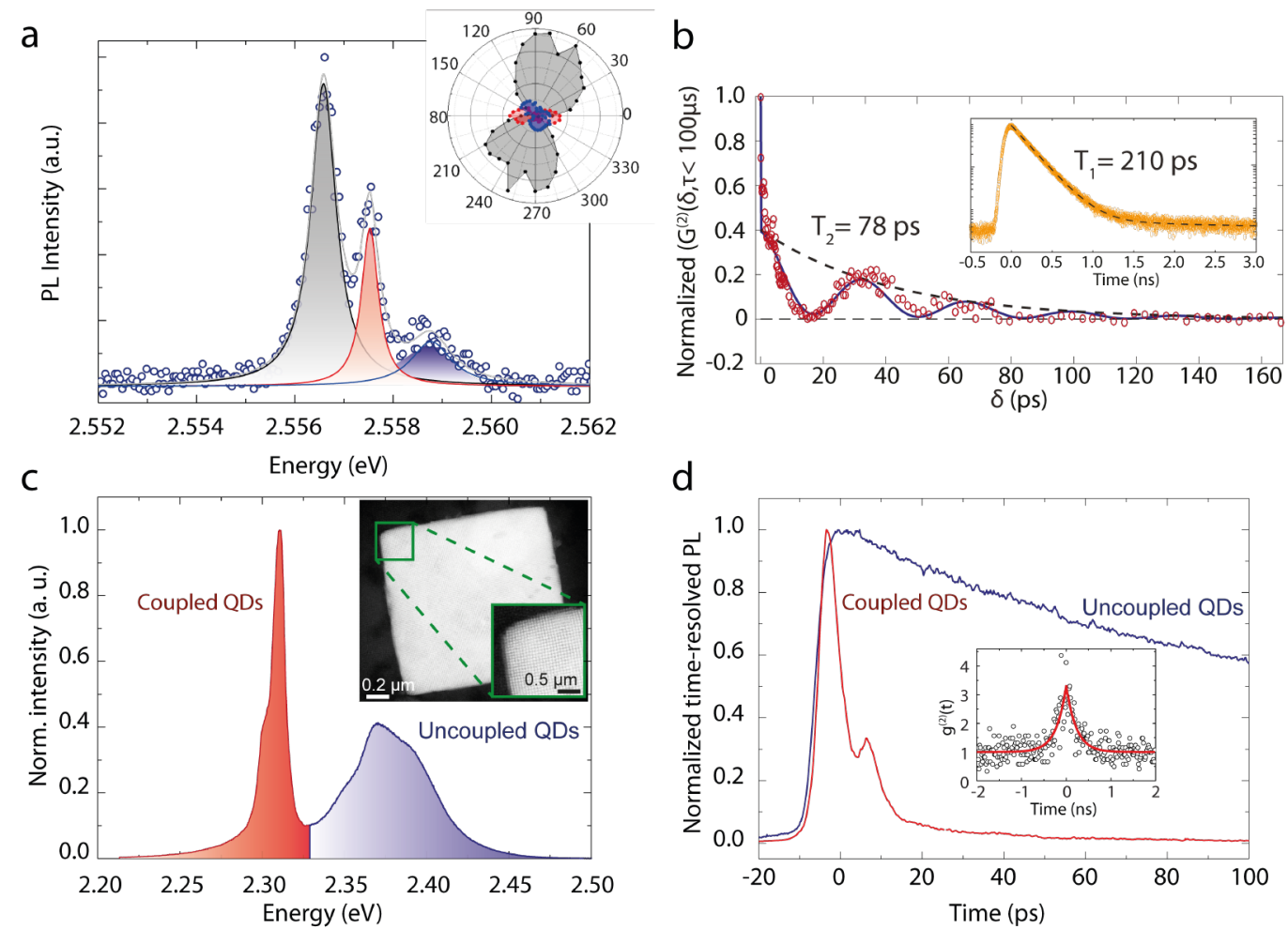

Figure 5: Superradiant emission: the case of perovskite quantum dots. (a) Single QD PL spectrum exhibiting bright triplet exciton states. (b) Measurements of the optical coherence times of single QDs. Coherence time on the order of $80 \mathrm{ps}$ is observed. The inset shows a time-resolved PL trace of a single QD, with typical radiative decay time of $200 \mathrm{ps}$, indicating the near transform-limited emission linewidths of single QDs. (c) PL spectrum from a single $\mathrm{CsPbBr}_{3} \mathrm{QD}$ superlattice at cryogenic temperatures. The inset shows the high-angle annular dark-field scanning transmission electron microscope (HAADF-STEM) image of a single superlattice of $\mathrm{CsPBr}_{3}$ QDs. (d) Time-resolved decay traces for uncoupled QDs (blue line) and coupled QDs (red line), which feature ultrafast decay (ca. 20 ps) and time oscillations at high excitation density. The inset shows the second order intensity-correlation measurements with bunching peak up to $\mathrm{g}^{(2)}(t=0) \sim 3$, indicating the strong collective nature of the photon-emission. (a) Adapted with permission from Reference 45. (b) Adapted with permission from Reference 46. (c, d) Adapted with permission from Reference 47.

Gabriele Rainò has been a senior research fellow at ETH Zurich since 2017. He received his $\mathrm{PhD}$ degree in innovative materials and technologies from the University of Salento, Italy, in 2008. In 2008, he joined IBM Research-Zürich as a postdoctoral researcher, studying organic-inorganic nanomaterials for integrated photonics. In 2011, he became a junior researcher at IBM Research-Zürich and 
began the exploration of colloidal nanomaterials as nonclassical light sources. His current research focuses on continuing the exploration of colloidal nanomaterials for optoelectronics and quantum applications. He has co-authored more than 45 publications in peer-reviewed journals and holds three patents. Rainò can be reached by email at rainog@ethz.ch.

Hendrik Utzat is a postdoctoral fellow at Stanford University, USA. He studied chemistry at RWTH Aachen University, Germany, and ETH Zürich, Switzerland and he received his $\mathrm{PhD}$ degree in physical chemistry from MIT in 2019. His current research focuses on solid-state emitters for quantum photonics and strong light-matter coupling. He a has published more than fifteen peer-reviewed publications in the areas of semiconducting polymers, solid-state quantum emitters, microfluidics, and spectroscopy. Utzat can be reached by email at hutzat@stanford.edu.

Moungi G. Bawendi is the Lester Wolfe Professor of Chemistry at the Massachusetts Institute of Technology (MIT). He received his AB degree from Harvard University in 1982, and his $\mathrm{PhD}$ degree in chemistry from the University of Chicago in 1988. He conducted postdoctoral research at Bell Laboratories. His research focuses on the technology of nanomaterials, including applications in light emission, energy harvesting, and biological imaging. He has published more than 500 scientific publications and holds more than 50 US patents. He is a fellow of the American Association for the Advancement of Science and the American Academy of Arts and Sciences, and is a member of the National Academy of Sciences. Bawendi can be reached by email at $\underline{\operatorname{mgb} @ \text { mit.edu. }}$

Maksym V. Kovalenko is a professor at ETH Zürich, Switzerland. He received his $\mathrm{PhD}$ degree in nanoscience and nanotechnology from Johannes Kepler University Linz, Austria, in 2007. He then joined the University of Chicago as a postdoctoral fellow, studying the surface chemistry and self-assembly of colloidal nanocrystals. In 2011, he joined ETH Zürich and Empa as a tenure-track assistant 
professor, an associate professor in 2017, and a full professor in 2020. His research interests include chemistry, physics, and applications of functional inorganic materials, including colloidal quantum dots, novel solid-state compounds, and materials for electrochemical energy storage. He has co-authored 250 publications and holds 11 patent applications. Kovalenko can be reached by email at mvkovalenko@ethz.ch.

\section{References}

1. The Nobel Prize in Physics 2000, https://www.nobelprize.org/prizes/physics/2000/summary/

2. The Nobel Prize in Physics 2014, https://www.nobelprize.org/prizes/physics/2014/summary/

3. MRS Bulletin 23 (2), (1998).

4. A. I. Ekimov, A. A. Onushchenko, Sov. Phys. Semicond. 16, 775-778 (1982).

5. A. L. Efros, A. L. Efros, Sov. Phys. Semicond. 16, 772-775 (1982).

6. $\quad$ MRS Bulletin 38 (9), (2013).

7. C. Adachi, M. A. Baldo, M. E. Thompson, S. R. Forrest, J. Appl. Phys. 90, 5048-5051 (2001).

8. M. Segal, M. Singh, K. Rivoire, S. Difley, T. Van Voorhis, M. A. Baldo, Nat. Mater. 6, 374-378 (2007).

9. Q. Zhang, B. Li, S. Huang, H. Nomura, H. Tanaka, C. Adachi, Nat. Photonics 8, 326-332 (2014).

10. Q. Wei, N. Fei, A. Islam, T. Lei, L. Hong, R. Peng, X. Fan, L. Chen, P. Gao, Z. Ge, Adv. Optical Mater. 6, 1800512 (2018).

11. Y. Zou, S. Gong, G. Xie, C. Yang, Adv. Optical Mater. 6, 1800568 (2018).

12. F. Guo, A. Karl, Q.-F. Xue, K. C. Tam, K. Forberich, C. J. Brabec, Light: Sci. Appl. 6, e17094 (2017).

13. X.-L. Chu, S. Götzinger, V. Sandoghdar, Nat. Photonics 11, 58-62 (2017).

14. P. Senellart, G. Solomon, A. White, Nat. Nanotechnol. 12, 1026-1039 (2017).

15. P. Siyushev, G. Stein, J. Wrachtrup, I. Gerhardt, Nature 509, 66-70 (2014).

16. T. A. Welton, Phys. Rev. 74, 1157-1167 (1948).

17. A. V. Kuhlmann, J. H. Prechtel, J. Houel, A. Ludwig, D. Reuter, A. D. Wieck, R. J. Warburton, Nat. Commun. 6, 8204 (2015).

18. D. Dufaker, L. O. Mereni, K. F. Karlsson, V. Dimastrodonato, G. Juska, P. O. Holtz, E. Pelucchi, Appl. Phys. Lett. 98, 251911 (2011).

19. O. Chen, J. Zhao, V. P. Chauhan, J. Cui, C. Wong, D. K. Harris, H. Wei, H.-S. Han, D. Fukumura, R. K. Jain, M. G. Bawendi, Nat. Mater. 12, 445-451 (2013). 
20. H. Utzat, K. E. Shulenberger, O. B. Achorn, M. Nasilowski, T. S. Sinclair, M. G. Bawendi, Nano Lett. 17, 6838-6846 (2017).

21. M. R. Salvador, M. W. Graham, G. D. Scholes, J. Chem. Phys. 125, 184709 (2006).

22. K. Cong, Q. Zhang, Y. Wang, G. Timothy Noe II, A. Belyanin, J. Kono, J. Opt. Soc. Am. B 33, 80-101 (2016).

23. R. H. Dicke, Phy. Rev. 93, 99-110 (1954).

24. F. Jahnke, C. Gies, M. Aßmann, M. Bayer, H. A. M. Leymann, A. Foerster, J. Wiersig, C. Schneider, M. Kamp, S. Höfling, Nat. Commun. 7, 11540 (2016).

25. N. Skribanowitz, I. P. Herman, J. C. MacGillivray, M. S. Feld, Phys. Rev. Lett. 30, 309-312 (1973).

26. K. Miyajima, Y. Kagotani, S. Saito, M. Ashida, T. Itoh, J. Physics: Condens. Matter 21, 195802 (2009).

27. M. S. Malcuit, J. J. Maki, D. J. Simkin, Boyd, W. Robert, Phys. Rev. Lett. 59, 1189-1192 (1987).

28. G. Timothy Noe II, J.-H. Kim, J. Lee, Y. Wang, A. K. Wójcik, S. A. McGill, D. H. Reitze, A. A. Belyanin, J. Kono, Nat. Phys. 8, 219-224 (2012).

29. F. Würthner, T. E. Kaiser, C. R. Saha-Möller, Angew. Chem. Int. Ed. 50, 3376-3410 (2011).

30. M. Kasha, Radiat. Res. 20, 55-70 (1963).

31. N. J. Hestand, F. C. Spano, Acc. Chem. Res. 50, 341-350 (2017).

32. J. Moll, S. Daehne, J. R. Durrant, D. A. Wiersma, J. Chem. Phys. 102, 63626370 (1995).

33. T. Stangl, P. Wilhelm, K. Remmerssen, S. Höger, J. Vogelsang, J. M. Lupton, Proc. Natl. Acad. Sci. 112, E5560-E5566 (2015).

34. C. Hettich, C. Schmitt, J. Zitzmann, S. Kühn, I. Gerhardt, V. Sandoghdar, Science 298, 385-389 (2002).

35. T. Heindel, A. Thoma, M. von Helversen, M. Schmidt, A. Schlehahn, M. Gschrey, P. Schnauber, J. H. Schulze, A. Strittmatter, J. Beyer, S. Rodt, A. Carmele, A. Knorr, S. Reitzenstein, Nat. Commun. 8, 14870 (2017).

36. J.-H. Kim, S. Aghaeimeibodi, C. J. K. Richardson, R. P. Leavitt, E. Waks, Nano Lett. 18, 4734-4740 (2018).

37. J. Q. Grim, A. S. Bracker, M. Zalalutdinov, S. G. Carter, A. C. Kozen, M. Kim, C. S. Kim, J. T. Mlack, M. Yakes, B. Lee, D. Gammon, Nat. Mater. 18, 963969 (2019).

38. L. Protesescu, S. Yakunin, M. I. Bodnarchuk, F. Krieg, R. Caputo, C. H. Hendon, R. X. Yang, A. Walsh, M. V. Kovalenko, Nano Lett. 15, 3692-3696 (2015).

39. J. Shamsi, A. S. Urban, M. Imran, L. De Trizio, L. Manna, Chem. Rev. 119, 3296-3348 (2019).

40. Q. A. Akkerman, G. Rainò, M. V. Kovalenko, L. Manna, Nat. Mater. 17, 394-405 (2018).

41. Y. Li, T. Ding, X. Luo, Z. Chen, X. Liu, X. Lu, K. Wu, Nano Res. 12, 619623 (2019).

42. R. E. Brandt, V. Stevanović, D. S. Ginley, T. Buonassisi, MRS Commun. 5, 265-275 (2015). 
43. M. Fu, P. Tamarat, H. Huang, J. Even, A. L. Rogach, B. Lounis, Nano Lett. 17, 2895-2901 (2017).

44. G. Rainò, G. Nedelcu, L. Protesescu, M. I. Bodnarchuk, M. V. Kovalenko, R. F. Mahrt, T. Stöferle, ACS Nano 10, 2485-2490 (2016).

45. M. A. Becker, R. Vaxenburg, G. Nedelcu, P. C. Sercel, A. Shabaev, M. J. Mehl, J. G. Michopoulos, S. G. Lambrakos, N. Bernstein, J. L. Lyons, T. Stöferle, R. F. Mahrt, M. V. Kovalenko, D. J. Norris, G. Rainò, A. L. Efros, Nature 553, 189-193 (2018).

46. H. Utzat, W. Sun, A. E. K. Kaplan, F. Krieg, M. Ginterseder, B. Spokoyny, N. D. Klein, K. E. Shulenberger, C. F. Perkinson, M. V. Kovalenko, M. G. Bawendi, Science 363, 1068-1072 (2019).

47. G. Rainò, M. A. Becker, M. I. Bodnarchuk, R. F. Mahrt, M. V. Kovalenko, T. Stöferle, Nature 563, 671-675 (2018).

48. C. Bradac, M. T. Johnsson, M. v. Breugel, B. Q. Baragiola, R. Martin, M. L. Juan, G. K. Brennen, T. Volz, Nat. Commun. 8, 1205 (2017).

49. $\quad$ MRS Bulletin 38 (2), (2013).

50. W. E. Moerner, Angew. Chem. Int. Ed. 54, 8067-8093 (2015).

51. C. S. Muñoz, E. del Valle, A. G. Tudela, K. Müller, S. Lichtmannecker, M. Kaniber, C. Tejedor, J. J. Finley, F. P. Laussy, Nat. Photonics 8, 550-555 (2014).

52. A. Aspuru-Guzik, P. Walther, Nat. Phys. 8, 285-291 (2012).

53. J. Huh, G. G. Guerreschi, B. Peropadre, J. R. McClean, A. Aspuru-Guzik, Nat. Photonics 9, 615-620 (2015). 